\title{
Investigation of the Nanostructured Semiconductor Metamaterials
}

\author{
Aleksej Trofimov, Tatjana Gric and Ortwin Hess \\ Additional information is available at the end of the chapter
}

http://dx.doi.org/10.5772/intechopen.72801

\begin{abstract}
The presence of electromagnetic waves on two-dimensional interfaces has been extensively studied over the last several decades. Surface plasmonic polariton (SPP), which normally exists at the interface between a noble metal and a dielectric, is treated as the most widely investigated surface wave. SPPs have promoted new applications in many fields such as microelectronics, photovoltaics, etc. Recently, it has been shown that by nanostructuring the metal surface, it is possible to modify the dispersion of SPPs in a prescribed manner. Herein, we demonstrate the existence of a new kind of surface wave between two anisotropic meta-materials. In contrast to extensively studied surface waves such as SPPs and Dyakonov waves, the surface waves supported by the nanostructured semiconductor metamaterial cross the light line, and a substantial portion at lower frequencies lies above the free-space light line. Consequently, the proposed structure will interact with the material via leaky waves.
\end{abstract}

Keywords: metamaterials, semiconductor, surface plasmon polaritons

\section{Introduction}

Plasmonics and the recent birth of metamaterials (MMs) [1-4] and transformation optics [5, 6] are currently opening a gateway to the development of a family of novel devices with unprecedented functionalities ranging from sub-wavelength plasmonic waveguides and optical nanoresonators [7] to superlenses, hyperlenses [8] and light concentrators [9]. Coupling between photons and surface plasmon polaritons (SPPs) $[10,11]$ is enabled by the periodically nanostructured metallic films allowing for exceptional and tunable optical properties determined by a combination of design geometry, the surrounding dielectric permittivity and the choice of metal $[12,13]$. SPPs have promoted new applications in many fields such as 
microelectronics [14], photovoltaics [15], near-field sensing [16], laser technology [17, 18], photonics [19], meta-materials design [2], high-order harmonics generation [20] or charged particle acceleration [21]. Recently, it has been shown that by nanostructuring the metal surface, it is possible to modify the dispersion of SPPs or excite the SPPs in a prescribed manner [22, 23].

The process of replacement of the uniaxial medium by a biaxial crystal [24], an indefinite medium [25] and a structurally chiral material [26] may enforce the presence of hybrid surface waves with some parallel characteristics. In the latter case, a methodology developed by Tamm [27] was adopted seeking to find a new type of surface wave, called as Dyakonov-Tamm wave, as it combines the features of Dyakonov surface waves (DSWs) and Tamm states. The use of structured materials with extreme anisotropy provided a fertile background aiming to increase the range of directions of DSWs substantially, as it is compared with the rather narrow range observed with natural birefringent materials [28]. Especially, outstanding results take place if the metallic nanoelements are employed to the anisotropic structures, as it occurs, for example, with a simple metal-dielectric multilayer, a case where the angular range may surpass half of a right angle [29]. The propagation length of these DSWs is drastically limited by the penetration depth inside the lossy MM [30] as it is caused by the specific damping capacity of metals.

The examination of two different interfaces, i.e. MM/dielectric and MM/TCO, is of the particular importance. Surface waves of different kinds, including DSWs along with traditional-like SPPs, are examined. Contrarily, the introduction of MM/TCO interface leads to a transformation of the traditional-like SPPs. As a consequence, the new types of surface waves are found.

Moreover, hyperbolic metamaterials, being special kind of anisotropic metamaterial with dielectric tenor elements having the mixed signs, have attracted growing attention due to their ability to support very large wave vectors. Their exotic features give rise to many intriguing applications, such as sub-wavelength imaging [31, 32] and hyper-lens [33, 34] that are infeasible with natural materials. In this paper, we demonstrate the existence of a new kind of surface wave between two anisotropic metamaterials. In contrast to extensively studied surface waves such as SPPs and Dyakonov waves, whose in-plane wave vector is greater than that of the bulk modes, the surface waves supported by the nanostructured semiconductor metamaterial cross the light line, and a substantial portion at lower frequencies lies above the free-space light line, which typically separates non-radiative (bound) and radiative (leaky) regions.

\section{Transparent conducting oxide (TCO) - dielectric composite heterostructure-based multilayer metamaterial}

The structure of the metamaterial (MM) is shown in Figure 1, where $d_{T C O}$ and $d_{d}$ represent the thicknesses of TCO and dielectric layers, respectively. All the involved media are nonmagnetic, so the magnetic permeability of every medium is the same as that of vacuum. In our numerical calculation, we use a semi-infinite $\mathrm{TCO} / \mathrm{PbS} \mathrm{MM}$ as an example to explore the dispersive features of hyperbolic surface plasmon polaritons (HSPPs), where $\varepsilon_{d}=18.8$ for PbS layers, and $\varepsilon_{T C O}$ is calculated using the parameters presented in [35]. 


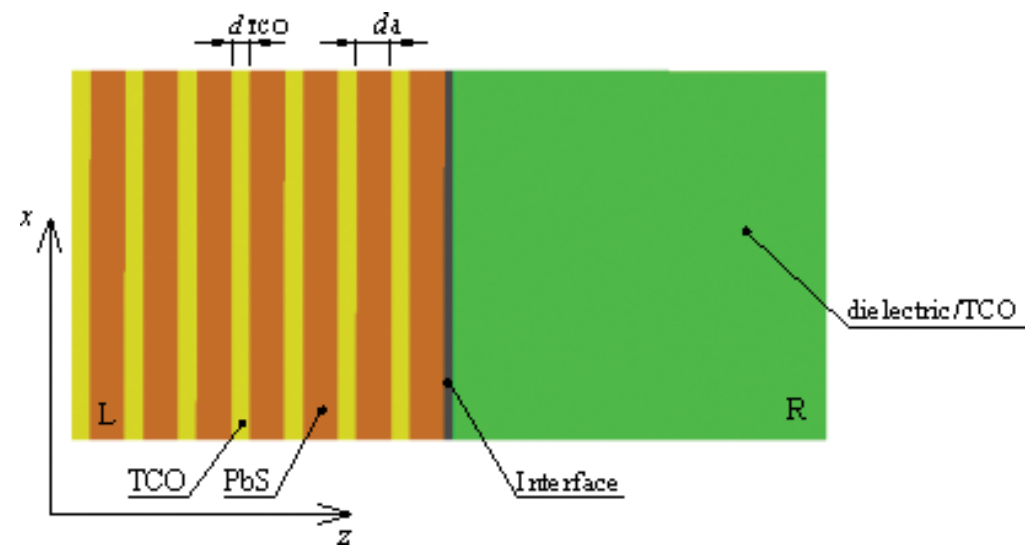

Figure 1. One-dimensional MM composed of alternating TCO and dielectric layers. The MM/dielectric $\left(\varepsilon_{R}=1\right.$ or $\left.\varepsilon_{R}=2.25\right)$ and $\mathrm{MM} / \mathrm{TCO}$ interfaces are considered.

Consequently, an example to estimate the limitation on the structure period under the effectivemedium theory (EMT) is considered. Proposing model, when the wavelength of radiation is much larger than the thickness of any layer, one can apply the effective-medium approach based on averaging the structure parameters.

First, the dispersion features of HSPPs are investigated. On the contrary to the approach presented in [36-38], the damping term in the TCO is not ignored in the process of analysing and calculating their dispersion properties. It is worth to mention that this particular MM is equivalent to a uniaxial-anisotropy effective medium, with its anisotropy axis (the optical axis) along the structure periodicity in the long-wavelength limit. Its effective permittivity tensor is written as

$$
\varepsilon=\varepsilon_{0}\left(\begin{array}{lll}
\varepsilon_{\|} & 0 & 0 \\
0 & \varepsilon_{\|} & 0 \\
0 & 0 & \varepsilon_{\perp}
\end{array}\right)
$$

in the principal-axis coordinate system. The principal values of the tensor are expressed with $[39,40]$

$$
\begin{gathered}
\varepsilon_{\|}=f_{T C O} \varepsilon_{T C O}+f_{d} \varepsilon_{d} \\
\varepsilon_{\perp}=\varepsilon_{T C O} \varepsilon_{d} /\left(f_{T C O} \varepsilon+f_{d} \varepsilon_{T C O}\right),
\end{gathered}
$$

where $f_{\mathrm{TCO}}=d_{\mathrm{TCO}} / L$ and $f_{d}=d_{d} / L, L=d_{d}+d_{\mathrm{TCO}}$ represent the TCO and dielectric filling ratios, respectively. It should be realized that MMs with a very large permittivity or a nearzero permittivity exhibit interesting properties [40]. While one may consider MMs with a very large permittivity as optical conductors, those with a near-zero permittivity can be used as optical insulators [40]. The zero point and divergence point of the principal values were also of 

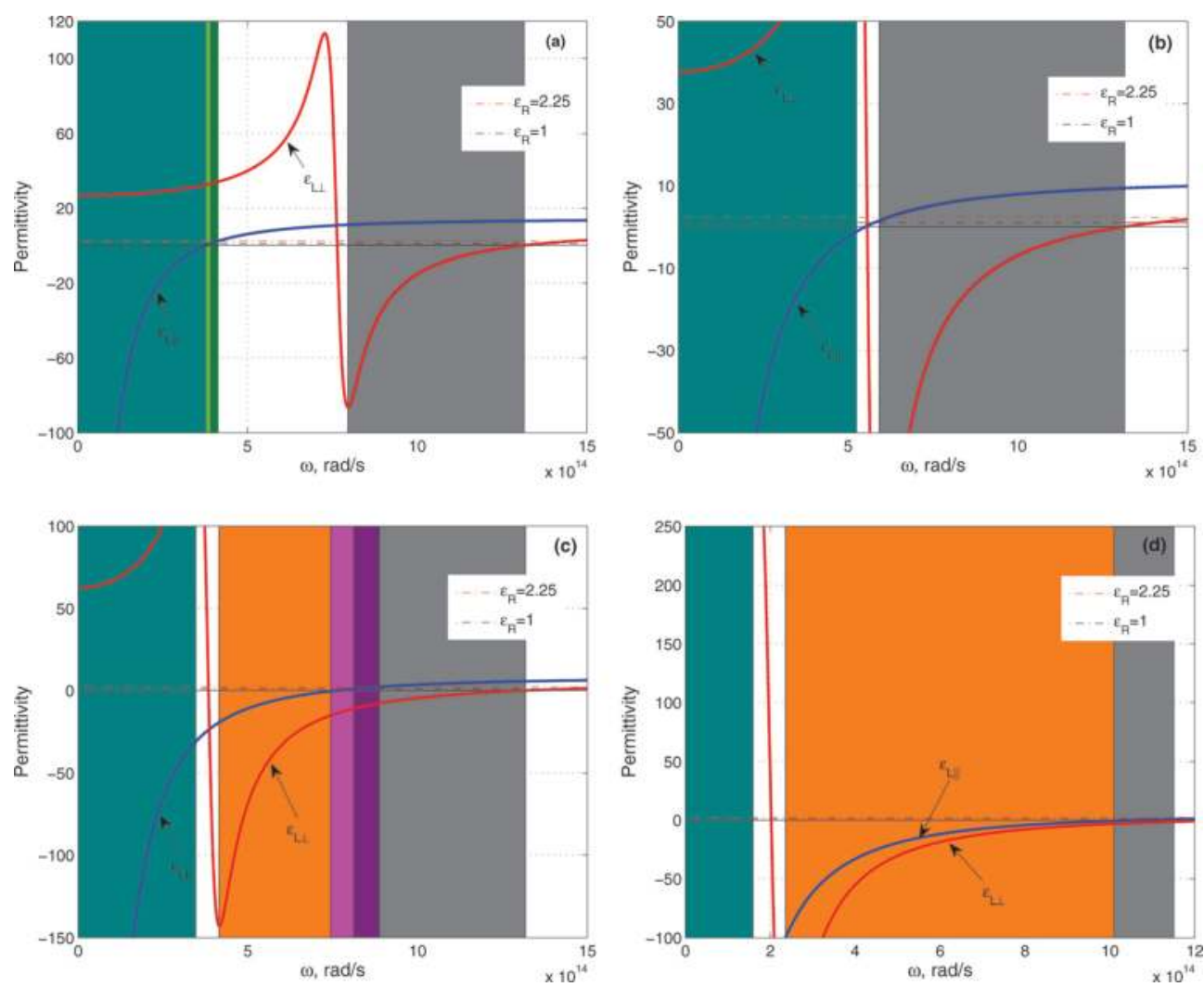

Figure 2. The principal values of effective permittivity and the different frequency regions for typical TCO-filling ratios corresponding to the $\mathrm{MM} /$ dielectric interface. $f_{\mathrm{TCO}}=0.3 \mathrm{in}(\mathrm{a}), f_{\mathrm{TCO}}=0.5 \mathrm{in}(\mathrm{b}), f_{\mathrm{TCO}}=0.7 \mathrm{in}(\mathrm{c})$ and $f_{\mathrm{TCO}}=0.9$ in (d).

the particular interest to discuss the dispersion features of SPPs [41]. The zero point and divergence point of $\varepsilon_{\perp}$ or $\varepsilon_{\|}$will be applied to discuss HSPPs.

Electric and magnetic fields' tangential components need to be evaluated at the interface in order to get metamaterial interface confined surface mode unique dispersion [42]:

$$
\beta=k \sqrt{\frac{\left(\varepsilon_{\|}^{R}-\varepsilon_{\|}^{L}\right) \varepsilon_{\perp}^{R} \varepsilon_{\perp}^{L}}{\varepsilon_{\perp}^{R} \varepsilon_{\|}^{R}-\varepsilon_{\perp}^{L} \varepsilon_{\|}^{L}}}
$$

where $k$ is the absolute value of wave vector in vacuum and $\beta$ is the component of the wave vector parallel to the interface.

It is interesting to notice that in the case of the MM interface, the obtained result for the dispersion is as follows: 

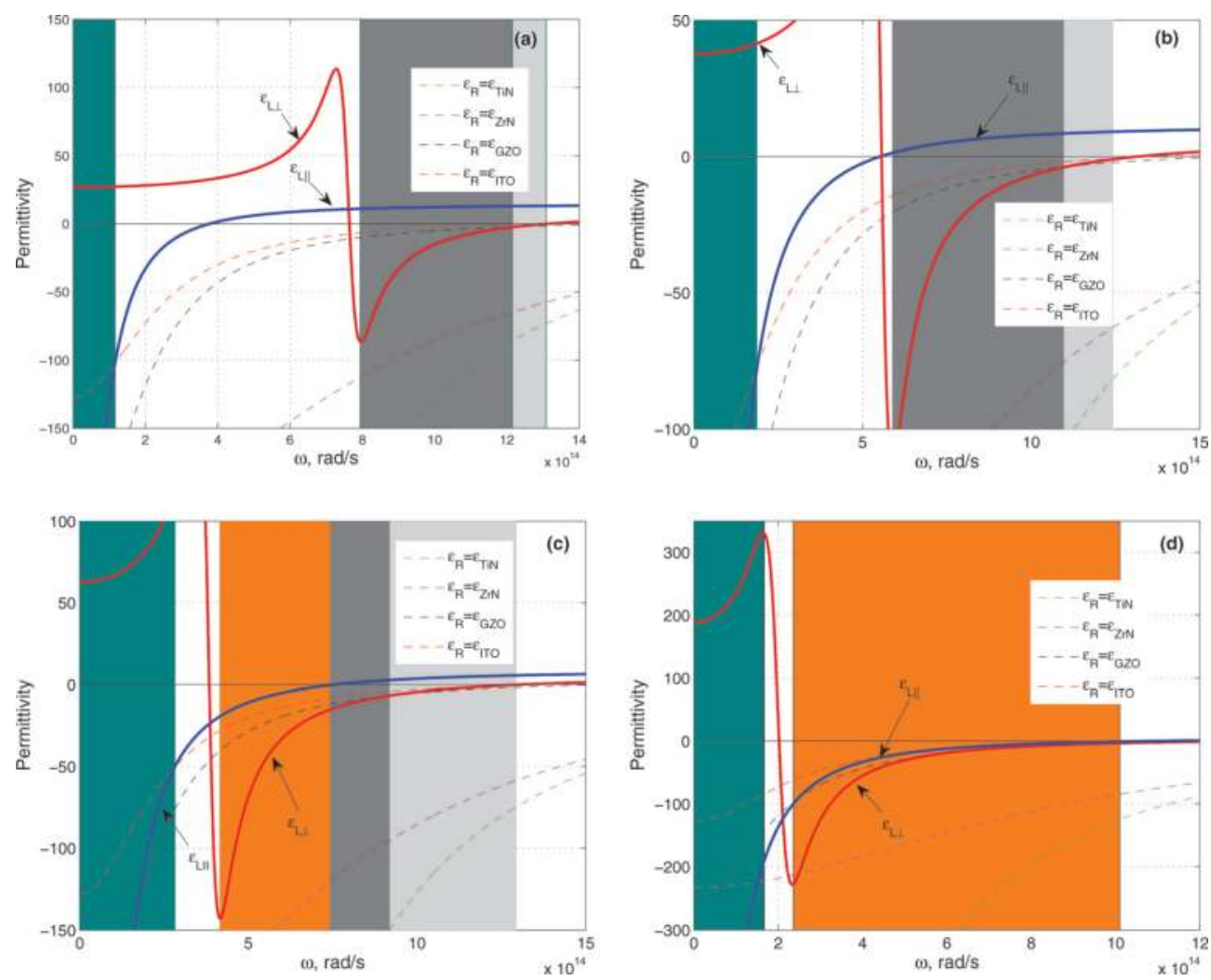

Figure 3. The principal values of effective permittivity and the different frequency regions for typical TCO-filling ratios, corresponding to the MM/TCO interface. $f_{\mathrm{TCO}}=0.3$ in (a), $f_{\mathrm{TCO}}=0.5$ in (b), $f_{\mathrm{TCO}}=0.7$ in (c) and $f_{\mathrm{TCO}}=0.9$ in (d).

$$
\beta=k\left(\frac{\varepsilon_{T C O} \varepsilon_{d} \varepsilon_{R}\left(d_{d}-\frac{d_{d} f_{T C O}}{f_{T C O}-1}\right)\left(\varepsilon_{d R}-\frac{a}{d_{d}-\frac{d_{d} f T C O}{f_{T C O}-1}}\right)}{\left(\varepsilon_{R}^{2}-\frac{\varepsilon_{T C O} \varepsilon_{d} a}{b}\right) b}\right)^{1 / 2},
$$

where $a=d_{d} \varepsilon_{d}-\frac{d_{d} \varepsilon_{T C O} f_{T C O}}{f_{T C O}-1}, b=d_{d} \varepsilon_{T C O}-\frac{d_{d} \varepsilon_{d} f_{T C O}}{f_{T C O}-1}, \varepsilon_{R}$ is the permittivity of the material at the right-hand side of the interface.

It is of particular interest to obtain the dispersion relation for the interface states in the effective media approach corresponding to the MM interface, having in mind that material at the righthand side is the same as employed in the MM. This dispersion relation reads

$$
\beta=k\left(\frac{\varepsilon_{T C O} \varepsilon_{d}}{\varepsilon_{T C O}+\varepsilon_{d}}\right)^{1 / 2}
$$



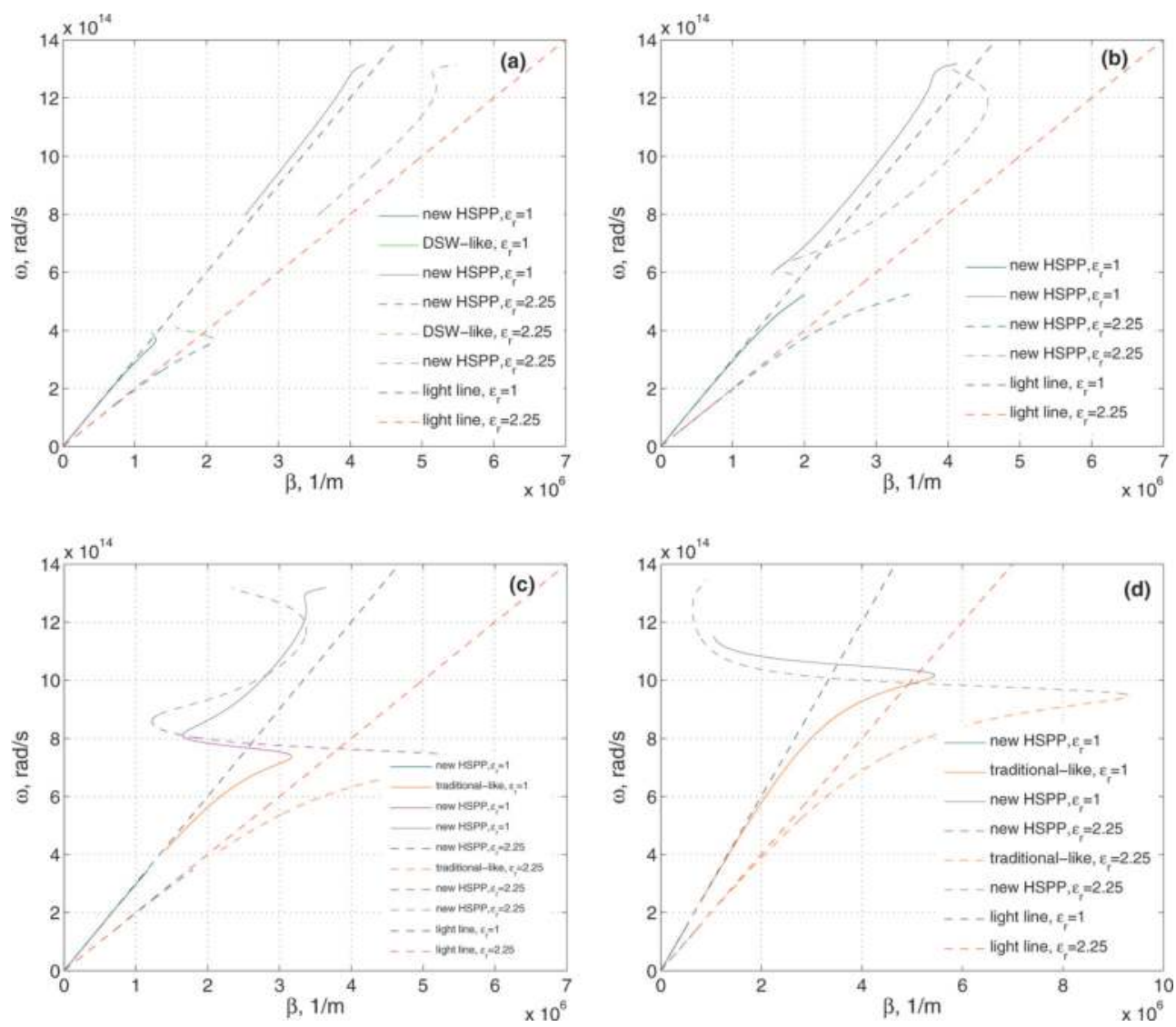

Figure 4. Dispersion curves of HSPPs for various TCO-filling ratios, i.e. $f_{\text {TCO }}=0.3$ in (a), $f_{\text {TCO }}=0.5$ in (b), $f_{\text {TCO }}=0.7$ in (c) and $f_{T C O}=0.9$ in $(\mathrm{d})$, corresponding to the $\mathrm{MM} /$ dielectric interface.

As a matter of fact, we obtain a surprising result: the dispersion of a (single) interface mode does not depend on the thicknesses of the layers, and it coincides with the dispersion of a conventional surface plasmon at metal-dielectric interface.

\subsection{The mode structure}

In the case of a spatially infinite anisotropic material, invariant in two directions, the electromagnetic wave dispersion can be plotted for both MM/dielectric and MM/TCO cases. Thus, herein, we present analysis performed after the homogenization of the MM corresponding to the MM/dielectric and MM/TCO interfaces. Doing so, in the numerical calculations, the semiinfinite $\mathrm{AZO} / \mathrm{PbS} \mathrm{MM}$ is taken as an example. We will first review the optical properties by depicting the curves of the principal values $\left(\varepsilon_{\perp}\right.$ and $\left.\varepsilon_{\|}\right)$and the dielectric constant $\left(\varepsilon_{R}\right)$ and then by distinguishing different frequency regions according to the properties of and relation 

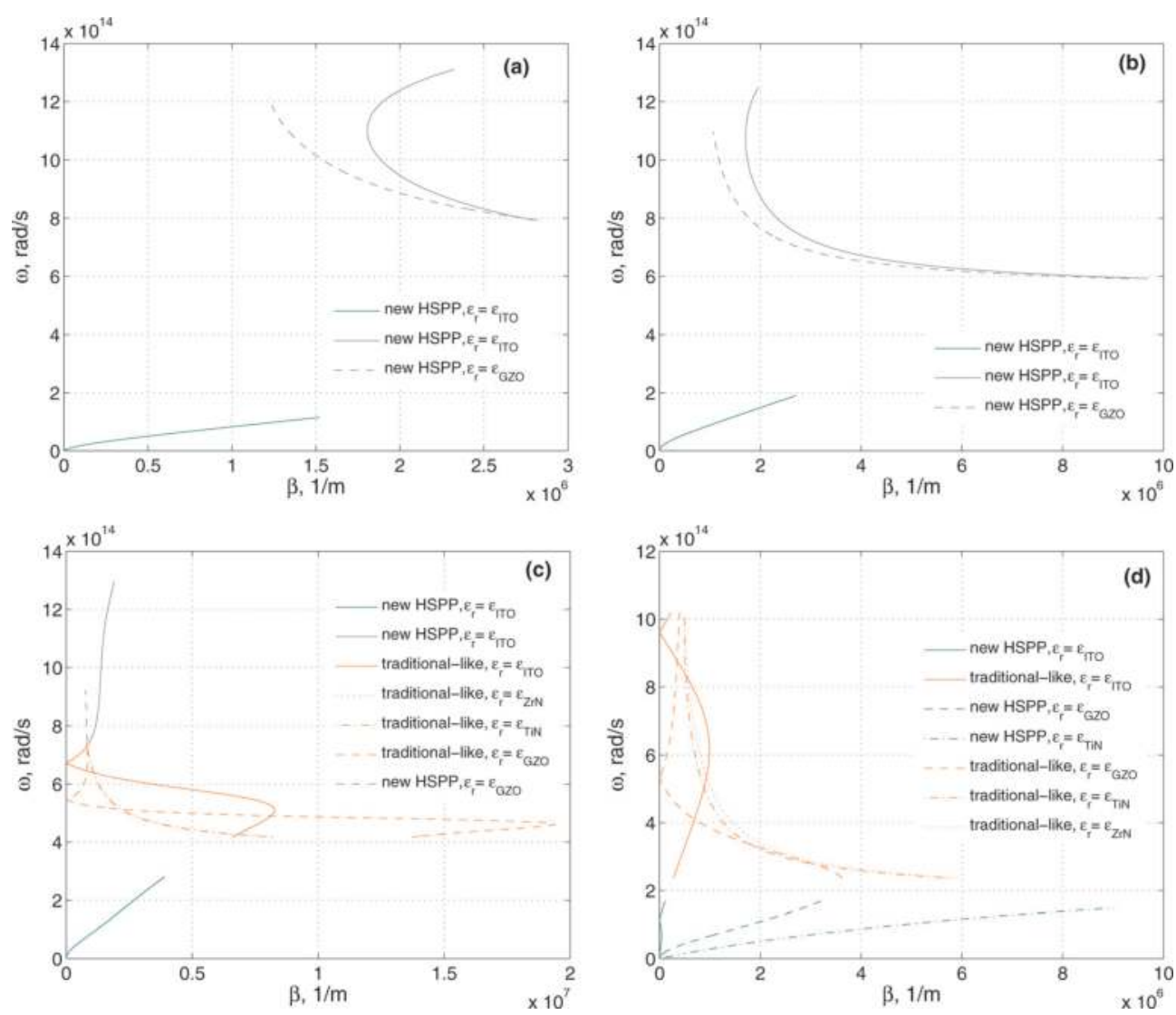

Figure 5. Dispersion curves of HSPPs for various TCO-filling ratios, i.e. $f_{\mathrm{TCO}}=0.3$ in (a), $f_{\mathrm{TCO}}=0.5$ in (b), $f_{\mathrm{TCO}}=0.7$ in (c) and $f_{\text {TCO }}=0.9$ in (d), corresponding to the MM/dielectric interface.

among $\varepsilon_{\perp}, \varepsilon_{\|}$and $\varepsilon_{R}$ as shown in Figures 2 and 3. $\varepsilon_{\perp}>\varepsilon_{R}>\varepsilon_{\|}$and $\varepsilon_{\|}<0$ in the cyan region, $\varepsilon_{\perp}>\varepsilon_{R}>\varepsilon_{\|}$in the green region, $\varepsilon_{\|}>\varepsilon_{R}>\varepsilon_{\perp}$ and $\varepsilon_{\perp}<0$ in the grey region, $\varepsilon_{R}>\varepsilon_{\|}>\varepsilon_{\perp}$ and $\varepsilon_{\perp}<0$ in the magenta region, and $\varepsilon_{\perp}<\varepsilon_{\|}<0$ in the orange region. The tunability of the effective parameters presented in Figures 2 and 3 has an effect on the dispersion curves of the HSPPs. As shown in Figures 2 and 3, the parameter that principally defines the tunability of the effective optical parameters of our metamaterial is the TCO-filling ratio, $f_{T C O}$. The dispersion curves are illustrated in Figures 4 and 5 for various TCO-filling ratios.

The curve colors correspond to different frequency regions. For $f_{T C O}=0.3$, there are three HSPPs belonging to three different kinds, respectively, in the case of $\mathrm{MM} /$ dielectric interface and two kinds of HSPPs in the case of MM/TCO interface. The upper waves exist in the gray region for MM/TCO case. Three exceptional cases related to $f_{\mathrm{TCO}}=0.5,0.7$ and 0.9 . In the case of $f_{\mathrm{TCO}}=0.5$, two kinds of HSPPs exist in two color regions, i.e. cyan and grey for MM/dielectric and MM/TCO interfaces. The upper short curves in the case of $f_{T C O}=0.7$ lie in the gray region, 

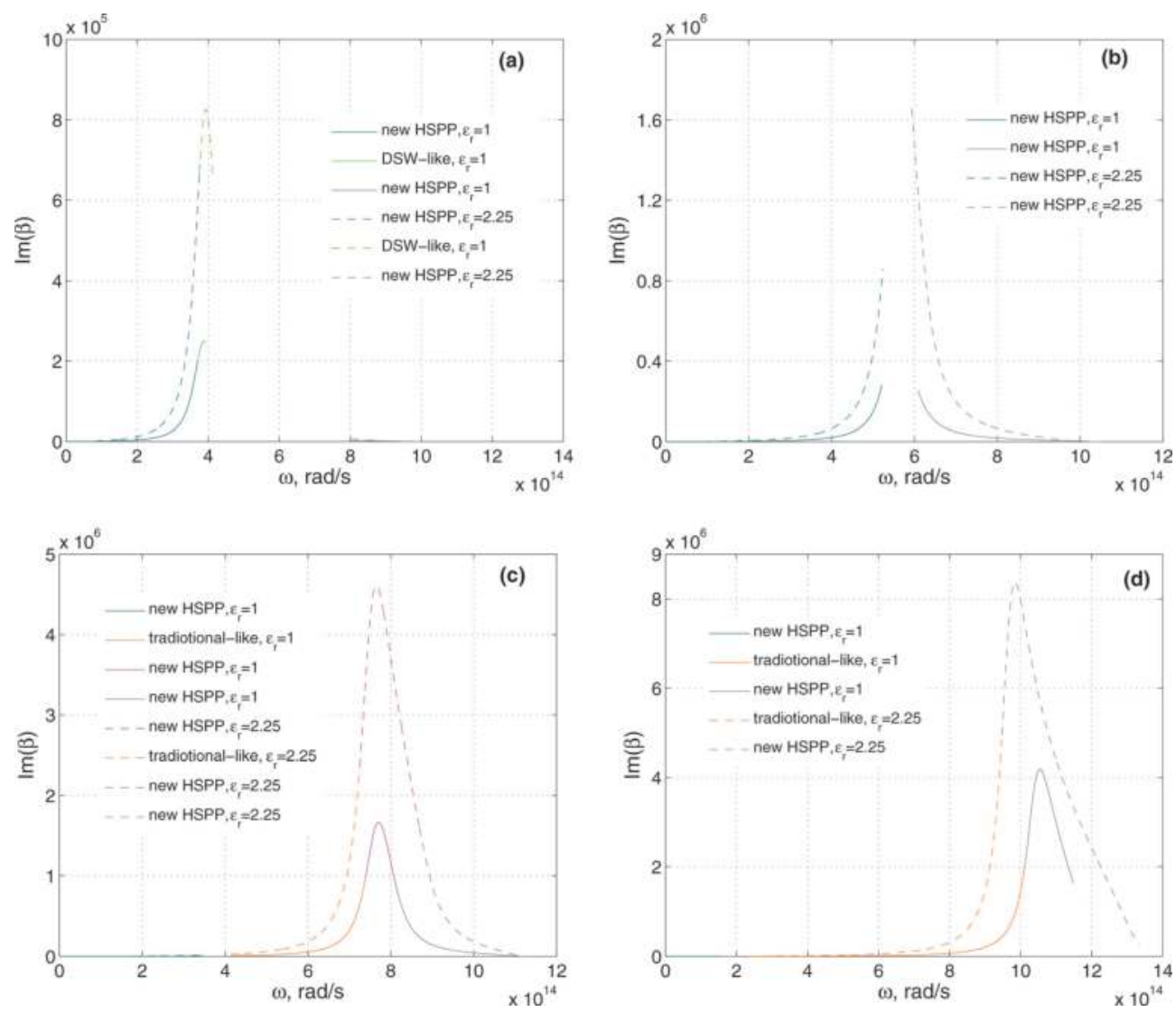

Figure 6. Absorption curves of HSPPs for various TCO-filling ratios, i.e. $f_{\mathrm{TCO}}=0.3$ in (a), $f_{\mathrm{TCO}}=0.5 \mathrm{in}(\mathrm{b}), f_{\mathrm{TCO}}=0.7 \mathrm{in}$ (c) and $f_{\mathrm{TCO}}=0.9 \mathrm{in}(\mathrm{d})$, corresponding to the $\mathrm{MM} /$ dielectric interface.

as illustrated in Figures 4 and 5. The other HSPPs lie in the orange region. The additional HSSPs lying in the magenta region correspond to the case of $\mathrm{MM} /$ dielectric interface. The extension of the magenta range for the case $\varepsilon_{R}=2.25$ is displayed by the dark-magenta color. In the case of $f_{T C O}=0.9$, two kinds of HSPPs are found lying in the orange and cyan regions for $\mathrm{MM} / \mathrm{TCO}$ case and three kinds of HSPPS lying in the cyan, orange and gray regions for MM/ dielectric case. Figure 4 also demonstrates that there always is one HSPP in the cyan region for various TCO-filling ratios in $\mathrm{MM}$ /dielectric case. It is worthwhile mentioning that the case $\varepsilon_{R}=\varepsilon_{I T O}$ also allows for the rich phenomenon as the HSPP always exists in the cyan region for various filling ratios (Figure 5).

Based on the necessary condition for the existence of the DSW [39], the HSPP in the green region (Figure 4) is similar to the DSW so that it should be called Dyakonov-like SPP [2] or the Dyakonov defined in [39]. Moreover, the frequency range of the DSW existence can be extended by replacing the material at the right-hand side of the interface with $\varepsilon_{R}=2.25$ 

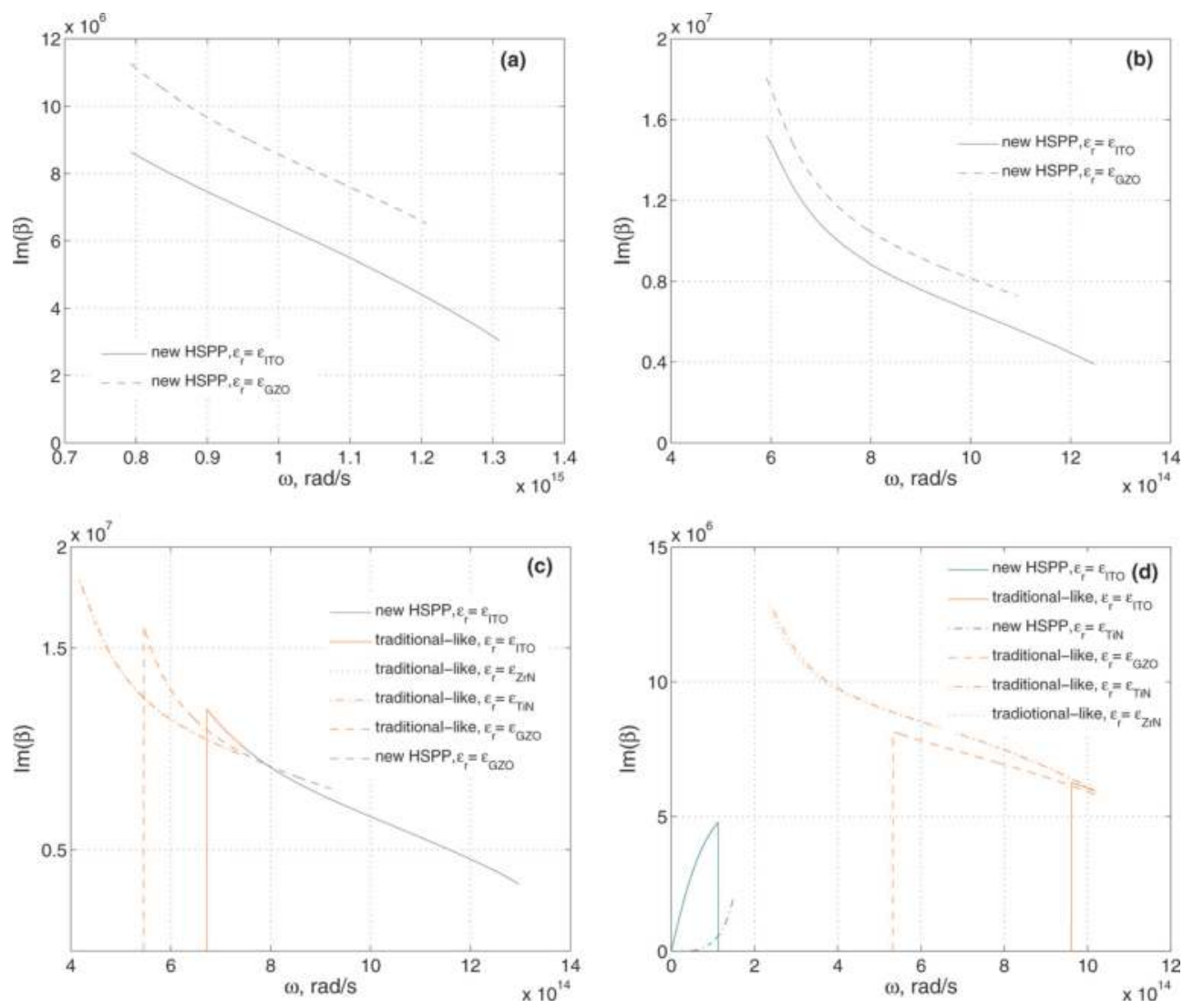

Figure 7. Absorption curves of HSPPs for various TCO-filling ratios, i.e. $f_{\mathrm{TCO}}=0.3 \mathrm{in}(\mathrm{a}), f_{\mathrm{TCO}}=0.5 \mathrm{in}(\mathrm{b}), f_{\mathrm{TCO}}=0.7 \mathrm{in}$ (c) and $f_{\mathrm{TCO}}=0.9 \mathrm{in}(\mathrm{d})$, corresponding to the MM/TCO interface.

(Figures 2 and 4). The former extension is demonstrated by the dark-green color in Figure 2. It is worthwhile mentioning that extension of the gray region in Figure 3 is possible due to the employment of ITO instead of GZO at the right-hand side of the interface (the extension of the gray range is depicted by the light-gray color). Due to the negative principal values of the effective permittivity in the orange region, the HSPP in this region is similar to the traditional SPP, so we should name it the traditional-like SPP. The others are new types of HSPPs. Thus, all the HSPPs are divided into five kinds, situated in the five color regions in Figures 4 and 5, respectively. In the case of $\mathrm{AZO} / \mathrm{PbS} \mathrm{MM}$ and air/dielectric interface, five types of the HSPPs exist, and only three are seen in the case of $\mathrm{AZO} / \mathrm{PbS} \mathrm{MM}$ and TCO interface.

As expected, the traditional-like SPP has a typical dispersion in the case of the MM/dielectric interface, lying to the right of the light line. At the same time, the degradation of the dispersion in the orange region takes place in the case of the MM/TCO interface. The dispersion properties can be tuned with the TCO-filling ratio of the MM realization. While for low TCO-filling ratio in the 
case of the MM/TCO interface, two types of the modes are always present, for higher TCO-filling ratio, the disappearance of modes in the gray region takes place (Figure 5(d)).

The complex mode structure (Figures 4 and 5) corresponding to either MM/dielectric or MM/ TCO interface emerges as a consequence of the confinement of plasmon polaritons in the direction perpendicular to the wave propagation. These electromagnetic surface waves arise via the coupling of the electromagnetic fields to oscillations of the conductor's electron plasma.

The imaginary parts of the wave vector (i.e. absorption) are plotted in Figures 6 and 7. It should be mentioned that negative values of the absorption in Figures 6 and 7 result from non-physical solutions of the dispersion equation and have been omitted in line with [43]. Taking advantage of the absorption resonances, one can show that the simple multilayer structures without possessing any periodic corrugation have the prospective to act as directive and monochromatic thermal sources [44].

\section{Nanostructured semiconductor metamaterial}

Another interesting MM structure depicted in Figure $\mathbf{8}$ is periodic stack of semiconductordielectric layers called hyperbolic metamaterial heterostructure.

The effective permittivity of the semiconductor (Si) can be calculated as follows:

$$
\varepsilon_{1,3}(\omega)=\varepsilon_{\infty}-\frac{\omega_{p}^{2}}{\omega^{2}+i \delta \omega^{\prime}}
$$

where $\varepsilon_{\infty}$ is the background permittivity and $\omega_{p}$ is the plasma frequency. The effective-medium approach [45] which is justified if the wavelength of the radiation considered is much larger

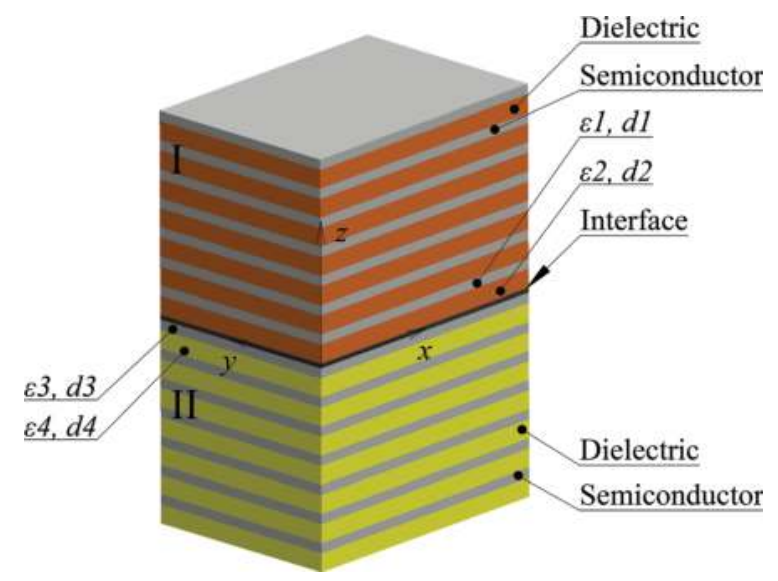

Figure 8. Geometry of the HMM. An interface separating two different semiconductor-dielectric-layered structures. Herein, indexes " 1 and 2" correspond to the semiconductor and dielectric layers correspondingly. 

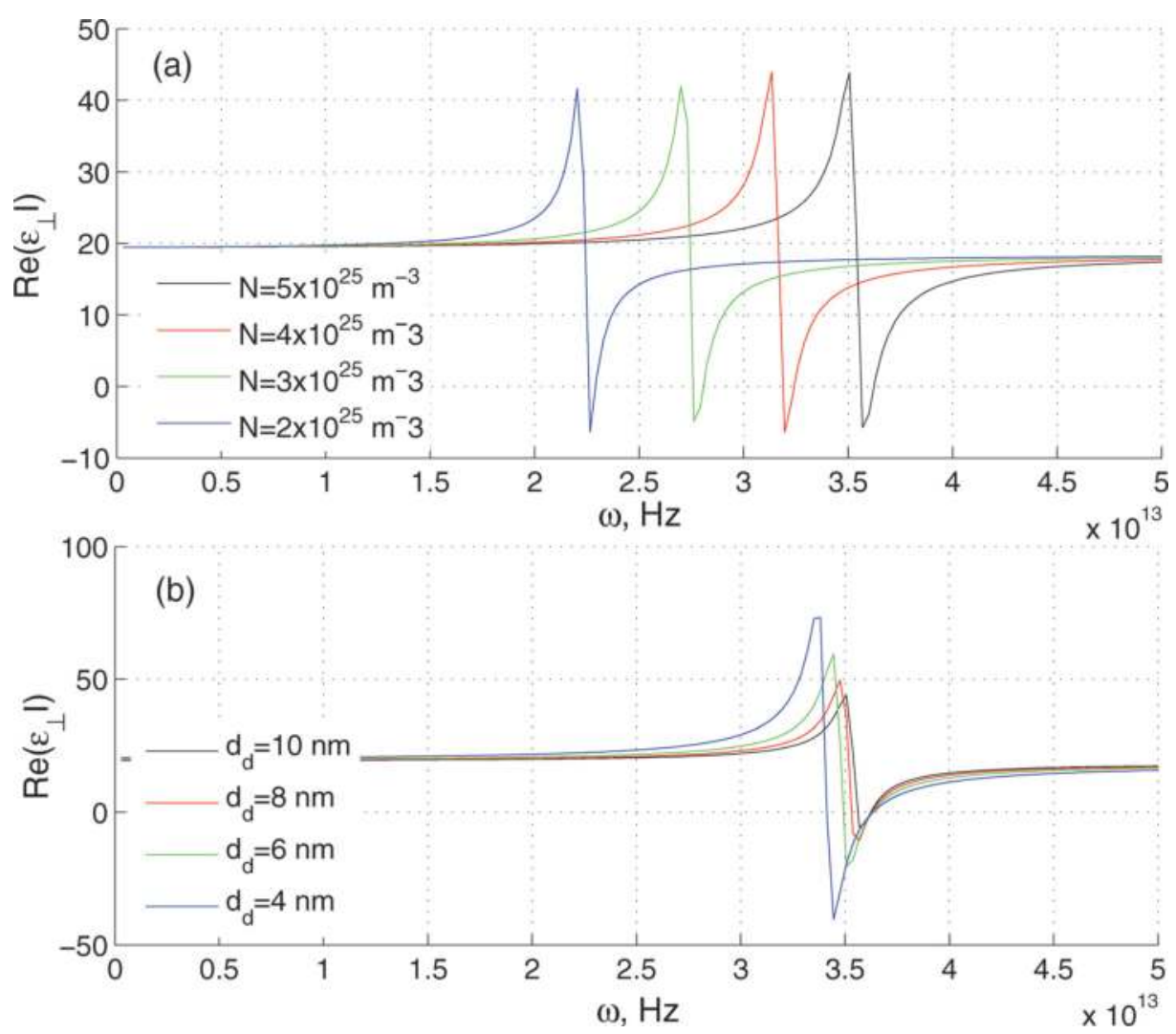

Figure 9. The influence of (a) doping level $\mathrm{N}$ and (b) thickness of dielectric $d_{d}$, on the real part of $\varepsilon_{\perp}$. $d_{d}=10 \mathrm{~nm}$ in (a) and $\mathrm{N}=5 \times 10^{25} \mathrm{~m}^{-3}$ in (b).

than the thickness of any layer is applied aiming to describe the optical response of such a system. The dispersion relation for the surface modes localized at the boundary separating two anisotropic media [42] is found by applying the appropriate boundary conditions, i.e. matching the tangential components of the electrical and magnetic fields at the interface. It is interesting to note that heavily doped silicon $\left(n>2.2 \times 10^{19} \mathrm{~cm}^{-3}\right)$ has been shown to exhibit metallic properties at terahertz frequencies $[46,47]$ and has the potential to replace metals in such applications [48]. The case of a heavy-doped $\mathrm{Si}$ is considered, assuming that the doping level is $N=5 \times 10^{19} \mathrm{~cm}^{-3}[49]$.

It should be mentioned that dramatic control of the frequency range of the surface wave existence is mostly concerned with the modifications of the permittivities and thicknesses of the layers [50] employed in the HMMs. To further study the surface waves, the tangential components of the electric and magnetic fields at the interface should be evaluated, and a single surface mode with the propagation constant should be obtained aiming to get the unique dispersion relation for the surface modes confined at the interface between two metamaterials [42]. 
Using the (4) formula, we can describe the case $\varepsilon_{1}=\varepsilon_{3}, \varepsilon_{2}=\varepsilon_{4}, d_{1} \neq d_{2} \neq d_{3} \neq d_{4}$ reveals the dispersion as follows:

$$
\beta=k \sqrt{\frac{\varepsilon_{1} \varepsilon_{2}}{\varepsilon_{1}+\varepsilon_{2}}} .
$$

The dispersion for the case of $\varepsilon_{1}=\varepsilon_{3}$ and $\varepsilon_{2} \neq \varepsilon_{4}, d_{1} \neq d_{2} \neq d_{3} \neq d_{4}$ is as follows:

$$
\beta=k \sqrt{\frac{\varepsilon_{1}^{2} \varepsilon_{2} \varepsilon_{4}\left(d_{1}+d_{2}\right)\left(d_{3}+d_{4}\right)\left(\frac{D}{d_{1}+d_{2}}-\frac{B}{d_{3}+d_{4}}\right)}{\left(\frac{\varepsilon_{1} \varepsilon_{2} D}{C}-\frac{\varepsilon_{1} \varepsilon_{4} B}{A}\right) C A}},
$$

where $A=d_{4} \varepsilon_{1}+d_{3} \varepsilon_{4}, B=d_{3} \varepsilon_{1}+d_{4} \varepsilon_{4}, C=d_{1} \varepsilon_{2}+d_{2} \varepsilon_{1}$ and $D=d_{1} \varepsilon_{1}+d_{2} \varepsilon_{2}$.

In the case of $\varepsilon_{1}=\varepsilon_{3}$ and $\varepsilon_{2} \neq \varepsilon_{4}, d_{1}=d_{3}$ and $d_{4}=d_{2}$ :
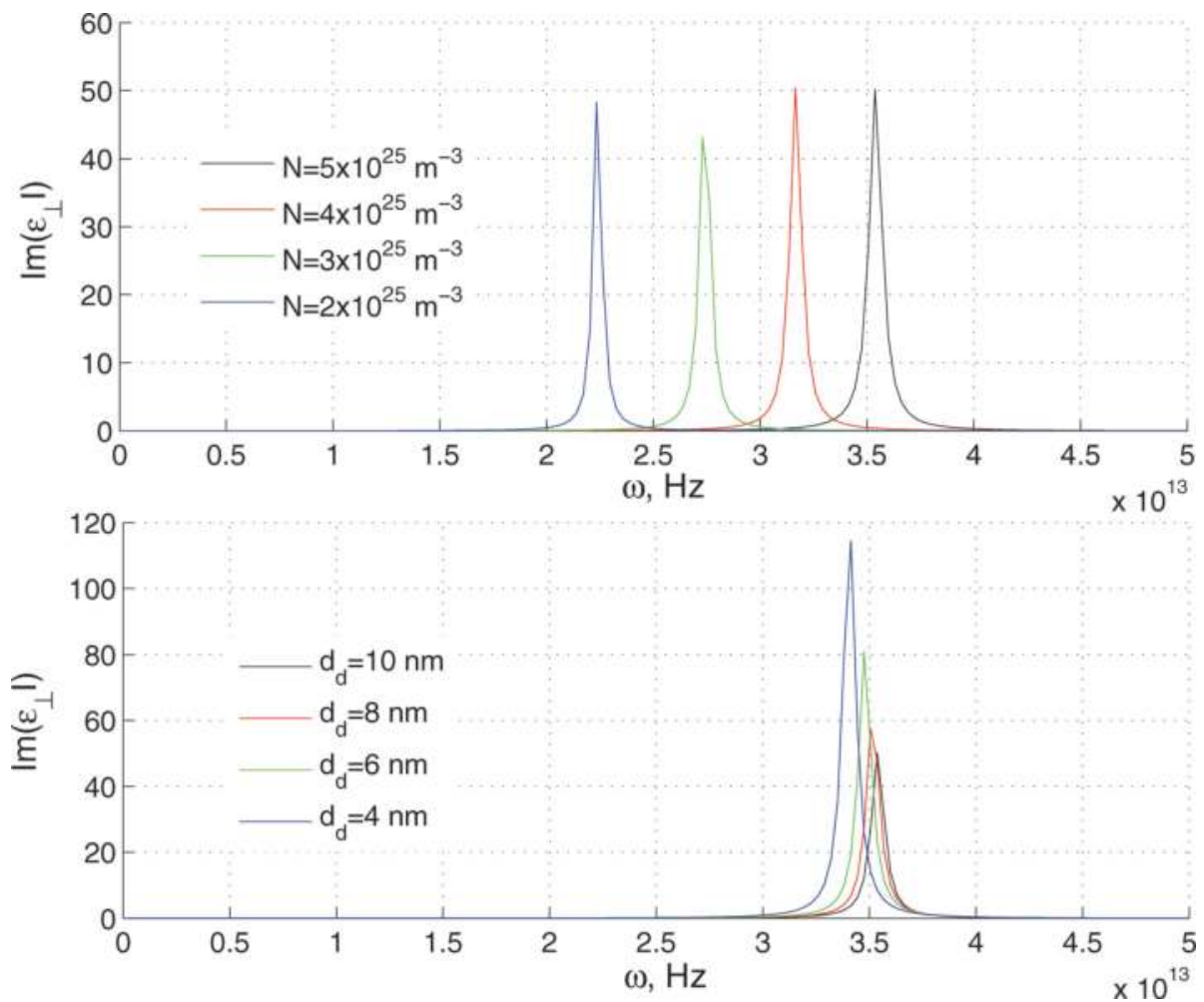

Figure 10. The influence of (a) doping level $\mathrm{N}$ and (b) thickness of dielectric $\mathrm{d}_{\mathrm{d}}$, on the imaginary part of $\varepsilon_{\perp}$. $\mathrm{d}_{\mathrm{d}}=10 \mathrm{~nm}$ in (a) and $\mathrm{N}=5 \times 10^{25} \mathrm{~m}^{-3}$ in (b). 


$$
\beta=k \sqrt{\frac{\varepsilon_{1} \varepsilon_{2} \varepsilon_{4}\left(d_{1}+d_{2}\right)}{d_{1} \varepsilon_{1}^{2}+d_{2} \varepsilon_{1} \varepsilon_{2}+d_{1} \varepsilon_{2} \varepsilon_{4}+d_{2} \varepsilon_{1} \varepsilon_{4}}}
$$

The permittivity spectra for the perpendicular components of the considered multilayer heterostructure are demonstrated in Figures 2(c), 3(a)-(c) and 9(a). Tuning the doping level of the semiconductor may open a gateway to the frequency control of the hyperbolic dispersion curve as shown in Figures 3(a), 4(a), 5(a) and 9(a). It is assumed that $d_{s}=0.35 \mathrm{~nm}$. PbS with relative permittivity $\varepsilon_{\mathrm{d}}=18.8$ and slab thickness $\mathrm{t}_{\mathrm{d}}=10 \mathrm{~nm}$ is chosen as the dielectric layer. It is clear that one has the potential to achieve the resonant behaviour of $\varepsilon_{\perp}$ by varying the doping level; moreover, the increase in the doping level causes a tuning of the resonant frequencies over the higher frequency range. Because of these attractive properties, our semiconductorbased layered structure has the great potential in the application as the building block for various HM-based optical devices.
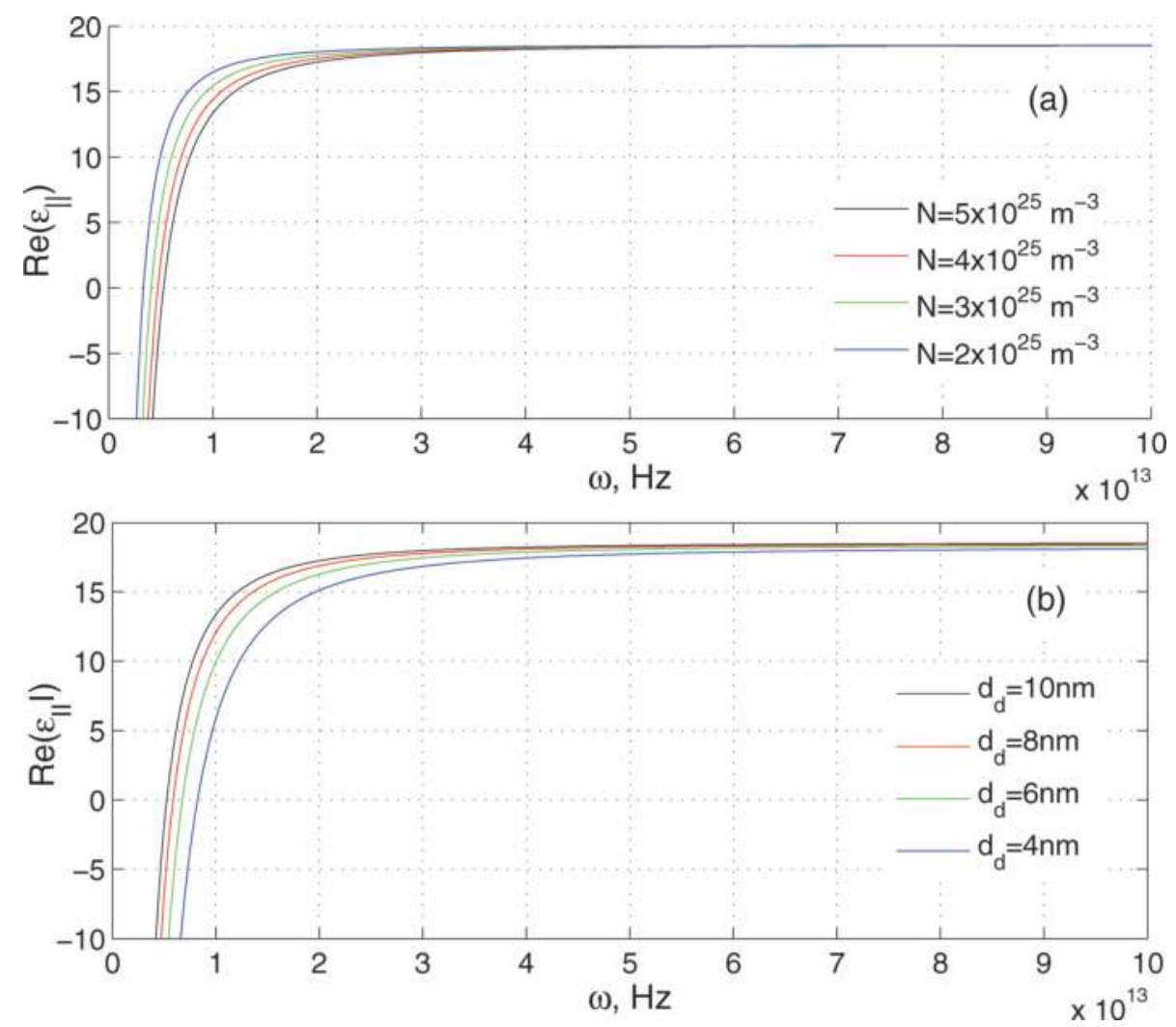

Figure 11. The influence of (a) doping level $\mathrm{N}$ and (b) thickness of dielectric $d_{d}$, on the real part of $\varepsilon_{\|} \cdot d_{d}=10 \mathrm{~nm}$ in (a) and $\mathrm{N}=5 \times 10^{25} \mathrm{~m}^{-3}$ in (b). 
Other than the doping level, the resonant behaviour of $\varepsilon_{\perp}$ was found to depend on the fill factions of the dielectric and semiconductor sheet, as shown in Figures $\mathbf{9 ( b )}$ and $\mathbf{3}(\mathbf{b})$. From Figure $9(\mathbf{b})$, the shift of the resonant frequency of $\varepsilon_{\perp}$ to the higher frequencies as the thickness $\mathrm{d}_{\mathrm{d}}$ is increased can be clearly distinguished.

\subsection{The mode structure}

Guided by the homogenization of two HMs, the computed dispersion curves are demonstrated. Thus, in Figure 13, the dispersion curves for the case $\varepsilon_{1}=\varepsilon_{3}, \varepsilon_{2}=\varepsilon_{4}$ and $d_{1} \neq d_{2} \neq d_{3} \neq d_{4}$ are shown. In this case, we deal with the boundary of two metamaterials with $d_{1}=0.35 \mathrm{~nm}$, $\mathrm{d}_{2}=10 \mathrm{~nm}, \mathrm{~d}_{3}=0.25 \mathrm{~nm}$ and $\mathrm{d}_{4}=10.1 \mathrm{~nm}$. Furthermore, due to the great interest in this case, Figure 13 refers to the dispersion of surface waves with the calculated effective parameters shown in Figures 9(a), 10(a), 11(a) and 12(a); the blue line is the free-space light line.

The frequency ranges of surface wave can be tuned by changing the doping level of silicon. As it is shown in Figure 9(a), doping level is correlated to the permittivity orthogonal component $\varepsilon_{\perp}$. Moreover, dispersion curves are shifted to the lower and higher frequencies with the
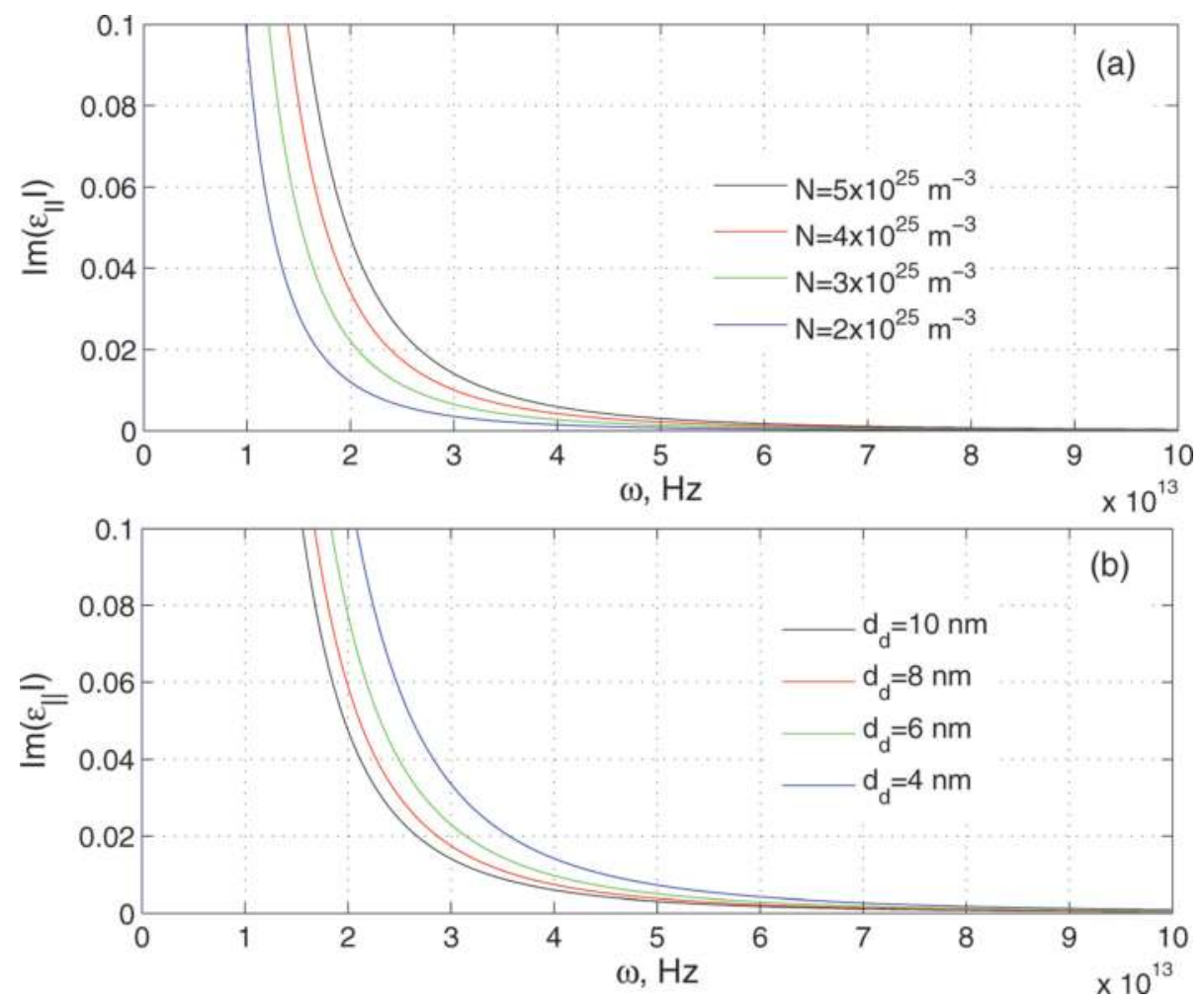

Figure 12. The influence of (a) doping level $\mathrm{N}$ and (b) thickness of dielectric $\mathrm{d}_{\mathrm{d}}$, on the imaginary part of $\varepsilon_{\|} \cdot \mathrm{d}_{\mathrm{d}}=10 \mathrm{~nm}$ in (a) and $\mathrm{N}=5 \times 10^{25} \mathrm{~m}^{-3}$ in (b). 

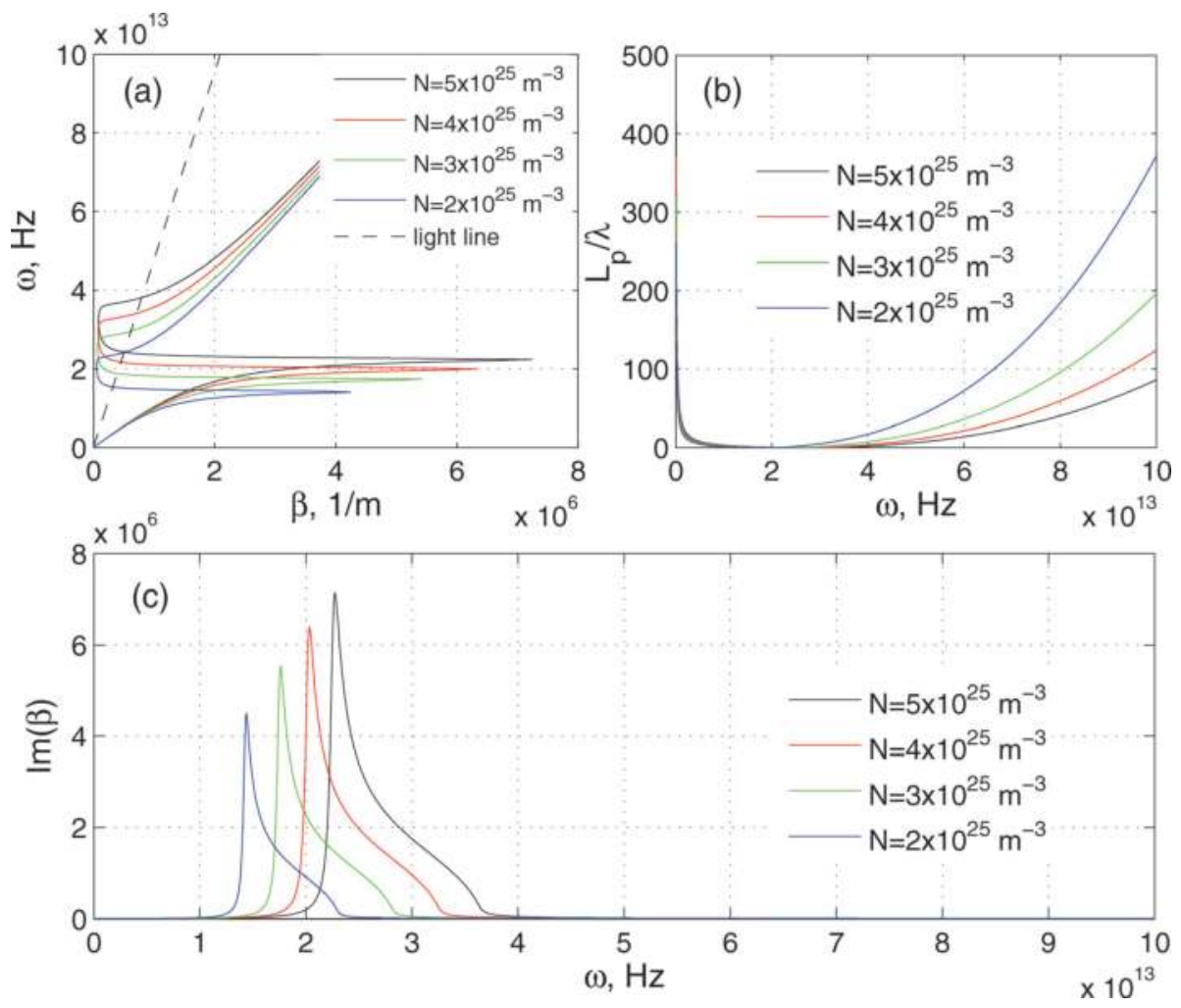

Figure 13. The dispersion of surface waves (a); propagation lengths (b) and absorption (c) at different doping levels of silicon, where $d_{d}=10 \mathrm{~nm}, \varepsilon_{1}=\varepsilon_{3}, \varepsilon_{2}=\varepsilon_{4}$ and $d_{1} \neq d_{2} \neq d_{3} \neq d_{4}$ and the blue line in (a) is the free-space light line.

decreases and increases in the doping level $\mathrm{N}$ accordingly. These tunability properties can be observed in Figure 13. Furthermore, this correlation can be used to engineer the metamaterial surface wave just by controlling silicon sheet doping level.

As the silicon is not modeled as lossless, $\beta$ is complex, leading to a finite propagation length (Eq. (2)), drawn in Figure 13(b). In Figure 13 the four modes $\left(\mathrm{N}=2 \times 10^{25} \mathrm{~m}^{-3}, \mathrm{~N}=3 \times 10^{25} \mathrm{~m}^{-3}\right.$, $\mathrm{N}=4 \times 10^{25} \mathrm{~m}^{-3}, \mathrm{~N}=5 \times 10^{25} \mathrm{~m}^{-3}$ ) always lie to the right side of the light line and remain nonradiative (bound) SP modes throughout the certain frequency range. All the considered cases are of particular interest due to the fact that their dispersion relations cross the light line and a significant portion at lower frequencies lies above the free-space light line, which usually splits up non-radiative (bound) and radiative (leaky) regions. For the bound modes, longer propagation lengths take place at lower frequencies owning the dispersion that is close to linear. Mode corresponding to the case $\mathrm{N}=4 \times 10^{25} \mathrm{~m}^{-3}$ possesses the longer propagation length than the mode corresponding to the case $\mathrm{N}=5 \times 10^{25} \mathrm{~m}^{-3}$.

The existence of the boundary modes associated with the second case under consideration, i.e. $\varepsilon_{1}=\varepsilon_{3}, \varepsilon_{2} \neq \varepsilon_{4}$ and $d_{1} \neq d_{2} \neq d_{3} \neq d_{4}$, is also of the particular importance. Thus, Figure 14 

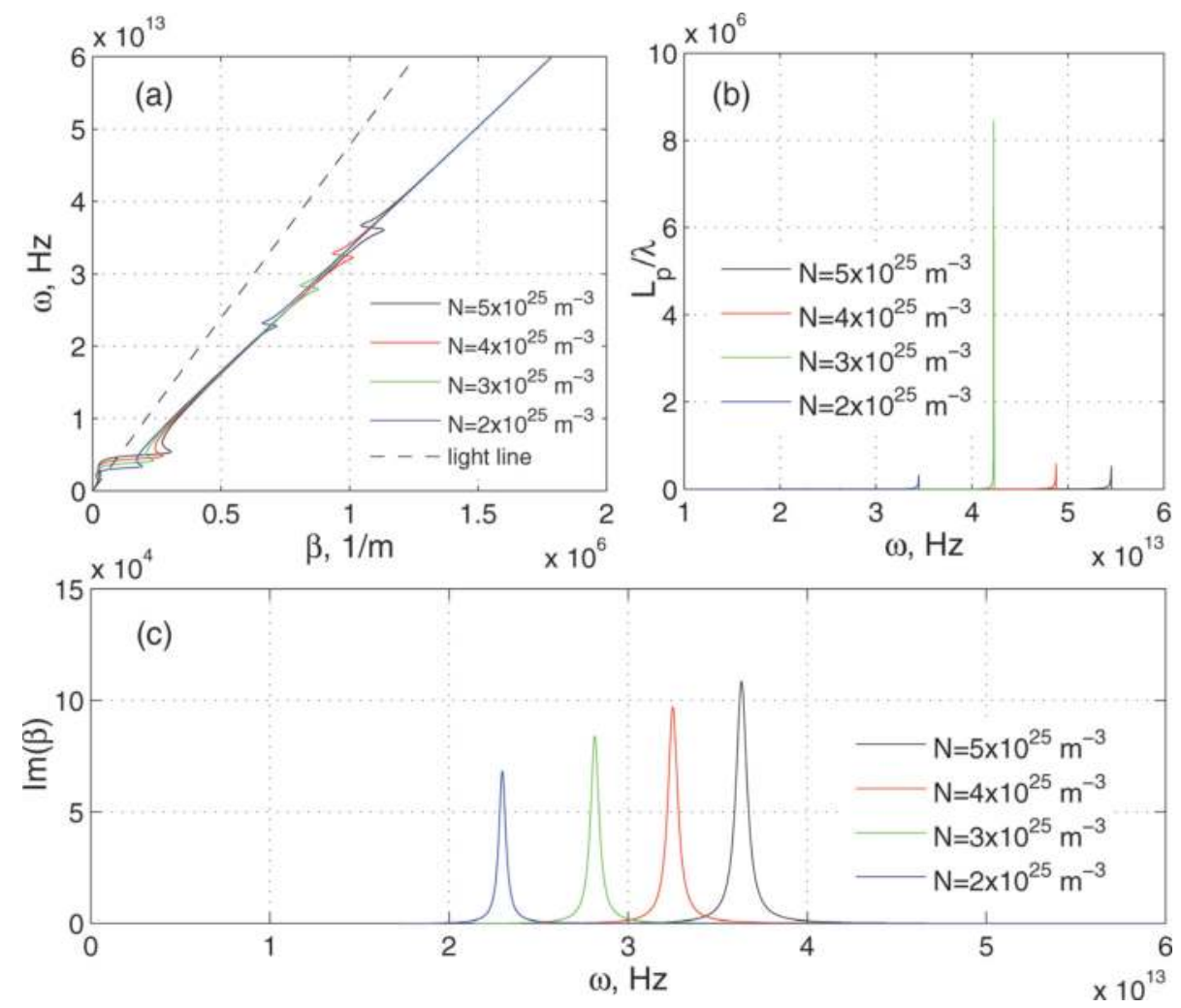

Figure 14. The dispersion of surface waves (a), propagation lengths (b) and absorption (c) at different doping levels, where $\mathrm{d}_{\mathrm{d}}=10 \mathrm{~nm}, \varepsilon_{1}=\varepsilon_{3}, \varepsilon_{2} \neq \varepsilon_{4}$ and $d_{1} \neq d_{2} \neq d_{3} \neq d_{4}$ and the blue line in (a) is the free-space light line.

tackles this problem by displaying four modes at the boundary of two different metamaterials with $\varepsilon_{4}=2.25$. As seen from Figure 14, the smallest asymptotic frequency corresponds to the case $\mathrm{N}=2 \times 10^{25} \mathrm{~m}^{-3}$.

In contrast to the previous case, we now discuss the instance denoted as $\varepsilon_{1}=\varepsilon_{3}, \varepsilon_{2} \neq \varepsilon_{4}$ and $d_{1}=d_{3}, d_{2}=d_{4}$ and shown by means of the dispersion diagrams of the TM modes. Thus, Figure 15 shows the dispersion curves of four different modes. The assessment and control of variation of the dielectric and semiconductor sheets' fill factors is of critical importance (Figure 15). First, the impact of the thickness of the dielectric $d_{d}$ on the dispersion curve (see Figure 15(a)) is considered. It is found that the upper limit moves to the higher frequencies as $d_{d}$ is increased. The former is consistent with the effect of $d_{d}$ on the frequency range of $\varepsilon_{\perp}$. The dependence of the frequency range of the surface waves existence on the thickness of dielectric stands for as the most critical feature of the HMs providing an unprecedented degree of freedom to control the surface wave at the near-infrared frequencies. In Figures 14(a) and 15(a), it is interesting to observe the Ferrell-Berreman modes which exist at energies near the ENZ of the hyperbolic metamaterial to the left of the light line [51-53]. 

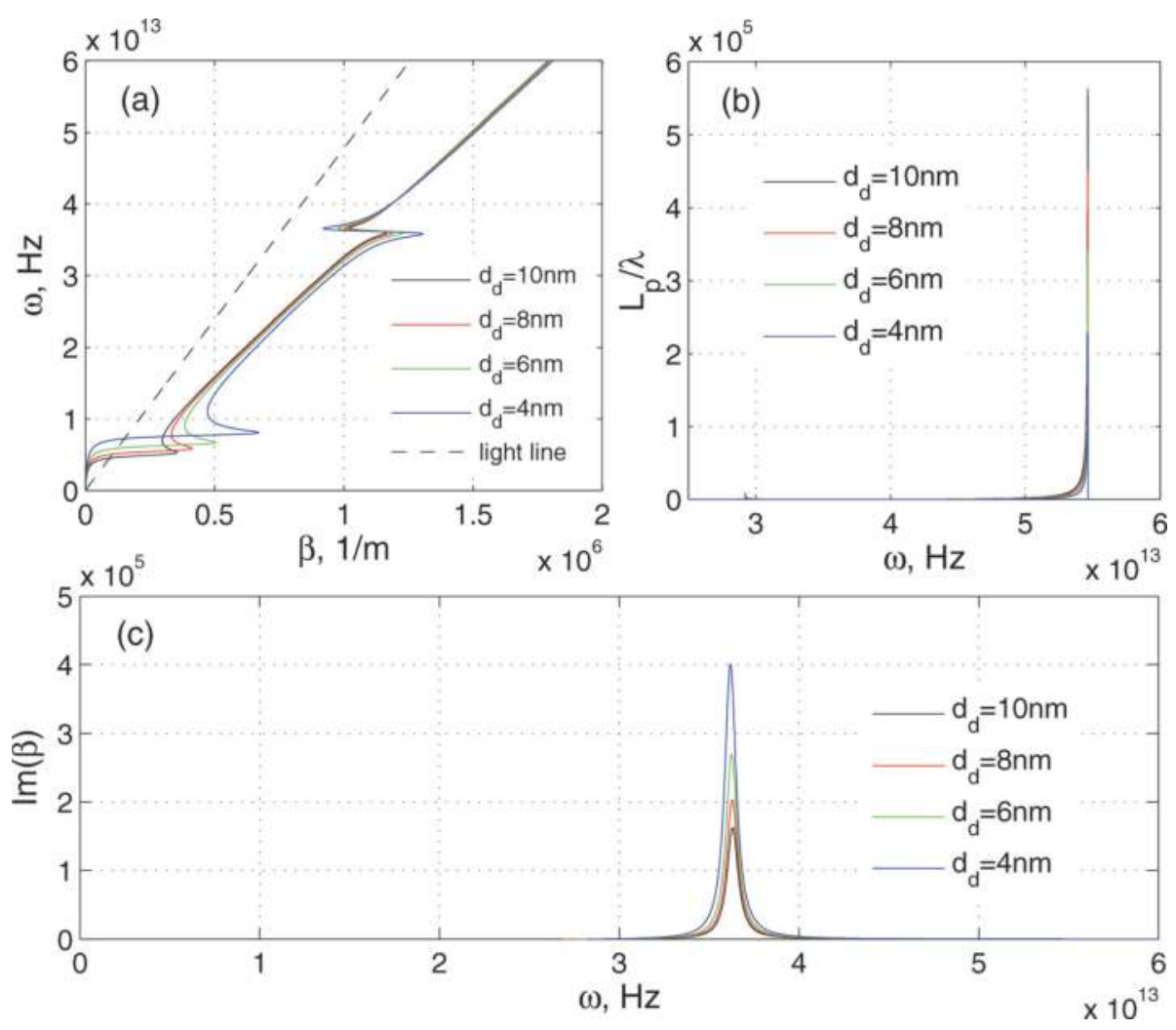

Figure 15. The dependences of dispersion of surface waves, propagation lengths and absorption on (a), (b) and (c) the thickness of dielectric $\mathrm{d}_{\mathrm{d}}$. Herein $\mathrm{N}=5 \times 10^{25} \mathrm{~m}^{-3}$.

\section{Conclusion}

During a study of the HSPPs in a one-dimensional TCO-dielectric MM, we can see that similar to graphene-dielectric MM [45], TCO-dielectric MM supports traditional-like SPPs having different patterns corresponding to two different interfaces. The dispersion equations of HSPPs are obtained based on the theoretical approach [42, 45]. Five kinds of HSPP, among which three kinds are new types of HSPPs and one is the Dyakonov-like SPP and another is the traditionallike SPP have been predicted. The existence of these HSPPs is dramatically influenced by the properties of and the relation among the principal values of the effective permittivity and the dielectric constant of the covering medium. It is worthwhile mentioning that the new types of the HSPPs arise because the principal values of the effective permittivity used in this chapter are functions of frequency and can be negative or positive. Moreover, it was demonstrated that used approach allows to predict surface mode with the dispersion that coincides with the dispersion of a surface plasmon at the boundary of two isotropic media corresponding to the MM interface if the material at the right-hand side is the same as employed in the MM. 
Moreover, a new kind of surface wave between two nanostructured semiconductor metamaterials was demonstrated. It is shown that the dispersion diagrams are sensitively dependent on the semiconductor parameters. These findings open the gateway towards potential applications in both classical and quantum optical signal communication and processing.

\section{Author details}

Aleksej Trofimov ${ }^{1 *}$, Tatjana Gric ${ }^{1}$ and Ortwin Hess ${ }^{2}$

*Address all correspondence to: aleksej.trofimov@vgtu.lt

1 Vilnius Gediminas Technical University, Vilnius, Lithuania

2 Imperial College London, London, UK

\section{References}

[1] Pendry J. Negative refraction makes a perfect lens. Physical Review Letters. 2000;85: 3966-3969

[2] Shalaev V. Optical negative-index metamaterials. Nature Photonics. 2007;1:41-48

[3] Shalaev V. Transforming light. Science. 2008;322:384-386

[4] Cai W, Shalaev V. Optical Metamaterials: Fundamentals and Applications. New York: Springer Verlag; 2009

[5] Pendry J, Schurig D, Smith D. Controlling electromagnetic fields. Science. 2006;312:17801782

[6] Schurig D, Mock J, Justice B, Cummer S, Pendry J, Starr A, Smith D. Metamaterial electromagnetic cloak at microwave frequencies. Science. 2006;314:977-980

[7] Lal S, Link S, Halas N. Nano-optics from sensing to waveguiding. Nature Photonics. 2007;1:641-648

[8] Salandrino A, Engheta N. Far-field subdiffraction optical microscopy using metamaterial crystals: Theory and simulations. Physical Review B. 2006;74:75103

[9] Kildishev A, Shalaev V. Engineering space for light via transformation optics. Optics Letters. 2008;33:43-45

[10] Tsakmakidis KL, Hermann C, Klaedtke A, Jamois C, Hess O. Surface plasmon polaritons in generalized slab heterostructures with negative permittivity and permeability. Physical Review B. 2006;73:085104 
[11] Ozbay E. Plasmonics: Merging photonics and electronics at nanoscale dimensions. Science. 2006;311:189-193

[12] Bouillard J-S, Vilain S, Dickson W, Zayats A. Hyperspectral imaging with scanning nearfield optical microscopy: Applications in plasmonics. Optics Express. 2010;18:16513

[13] Dickson W, Wurtz GA, Evans PR, Pollard RJ, Zayats AV. Electronically controlled surface plasmon dispersion and optical transmission through metallic hole arrays using liquid crystal. Nano Letters. 2008;8:281

[14] MacDonald KF, Samson ZL, Stockman MI, Zheludev NI. Ultrafast active plasmonics. Nature Photonics. 2009;3(1):55-58

[15] Atwater HA, Polman A. Plasmonics for improved photovoltaic devices. Nature Materials. 2010;9:205-213

[16] Kabashin A, Evans P, Pastkovsky S, Hendren W, Wurtz G, Atkinson R, Pollard R, Podolskiy V, Zayats A. Plasmonic nanorod metamaterial for biosensing. Nature Materials. 2009;8:867-871

[17] Berini P, De Leon I. Surface plasmon-polariton amplifiers and lasers. Nature Photonics. 2011;6(1):16-24

[18] Park I-Y, Kim S, Choi J, Lee D-H, Kim Y-J, Kling MF, Stockman MI, Kim S-W. Plasmonic generation of ultrashort extreme-ultraviolet light pulses. Nature Photonics. 2011;5(11): $677-681$

[19] Liu H, Lalanne P. Microscopic theory of the extraordinary optical transmission. Nature. 2008;452(7188):728-731

[20] Kim S, Jin J, Kim Y-J, Park I-Y, Kim Y, Kim S-W. High-harmonic generation by resonant plasmon field enhancement. Nature. 2008;453(7196):757-760

[21] Purvis MA, Shlyaptsev VN, Hollinger R, Bargsten C, Pukhov A, Prieto A, Wang Y, Luther BM, Yin L, Wang S, Rocca JJ. Relativistic plasma nanophotonics for ultrahigh energy density physics. Nature Photonics. 2013;7(10):796-800

[22] Williams CR, Andrews SR, Maier SA, Fernández-Domínguez AI, Martín-Moreno L, García-Vidal FJ. Highly confined guiding of terahertz surface plasmon polaritons on structured metal surfaces. Nature Photonics. 2008;2:175-179

[23] Huang L, Chen X, Bai B, Tan Q, Jin G, et al. Helicity dependent directional surface plasmon polariton excitation using a metasurface with interfacial phase discontinuity. Light: Science \& Applications. 2013;2:e70

[24] Liscidini M, Sipe JE. Quasiguided surface plasmon excitations in anisotropic materials. Physical Review B. 2010;81:115335

[25] Zapata-Rodríguez CJ, Miret JJ, Vuković S, Belić MR. Engineered surface waves in hyperbolic metamaterials. Optics Express. 2013;21:19113-19127 
[26] Gao J, Lakhtakia A, Lei M. Dyakonov-tamm waves guided by the interface between two structurally chiral materials that differ only in handedness. Physical Review A. 2010;81: 013801

[27] Tamm I. Possible type of electron binding on crystal surfaces. Zeitschrift für Physik. 1932;76:849-850

[28] Polo JA Jr, Nelatury SR, Lakhtakia A. Controlling hybrid-polarization surface plasmon polaritons in dielectric-transparent conducting oxides metamaterials via their effective properties. Journal of Nanophotonics. 2007;1:013501

[29] Vuković SM, Miret JJ, Zapata-Rodríguez CJ, Jakšić Z. Oblique surface waves at an interface between a metal-dielectric superlattice and an isotropic dielectric. Physica Scripta. 2012;2012(T149):014041

[30] Zapata-Rodríguez CJ, Miret JJ, Sorni JA, Vuković S. Propagation of dyakonon wavepackets at the boundary of metallodielectric lattices. IEEE Journal of Selected Topics in Quantum Electronics. 2013;19:4601408

[31] Salandrino A, Engheta N. Far-field subdiffraction optical microscopy using metamaterial crystals: Theory and simulations. Physical Review B. 2006;74:075103

[32] Shvets G, Trendafilov S, Pendry JB, Sarychev A. Guiding, focusing, and sensing on the subwavelength scale using metallic wire arrays. Physical Review Letters. 2007;99:053903

[33] Liu Z, Lee H, Xiong Y, Sun C, Zhang X. Far-field optical hyperlens magnifying subdiffraction-limited objects. Science. 2007;315:1686

[34] Rho J, Ye Z, Xiong Y, Yin X, Liu Z, et al. Spherical hyperlens for two-dimensional subdiffractional imaging at visible frequencies. Nature Communications. 2010;1:143

[35] Naik GV, Shalaev VM, Boltasseva A. Alternative plasmonic materials: Beyond gold and silver. Advanced Materials. 2013;25:3264-3294

[36] Cattom MG, Tilley DR. Introduction to Surface and Superlattice Excitations. Bristol: IOP Publishing; 2005, Ch. 8-9

[37] Li R, Cheng C, Ren F-F, Chen J, Fan Y-X, Ding J, Wang H-T. Hybridized surface plasmon polaritons at an interface between a metal and a uniaxial crystal. Applied Physics Letters. 2008;92:141115

[38] Sorni JA, Naserpour M, Zapata-Rodriguez CJ, Miret JJ. Dyakonov surface waves in lossy metamaterials. Optics Communications. 2015;355:251

[39] Takayama O, Artigas D, Torner L. Practical dyakonons. Optics Letters. 2012;37:4311

[40] Orlov AA, Voroshilov PM, Belov PA, Kivshar YS. Engineered optical nonlocality in nanostructured metamaterials. Physical Review B. 2011;84:045424

[41] Zhang Y-L, Zhang Q, Wang X-Z. Extraordinary surface polaritons in obliquely truncated dielectric/metal metamaterials. Journal of the Optical Society of America B. 2016;33:543 
[42] Iorsh I, Orlov A, Belov P, Kivshar Y. Interface modes in nanostructured metal-dielectric metamaterials. Applied Physics Letters. 2011;99(15):151914

[43] Ling RT, Scholler JD, Ufimtsev PY. The propagation and excitation of surface waves in an absorbing layer. Progress in Electromagnetics Research. 1998;19:49-91

[44] Campione S, Marquier F, Hugonin J-P, Ellis AR, Klem JF, Sinclair MB, Luk TS. Directional and monochromatic thermal emitter from epsilon-near-zero conditions in semiconductor hyperbolic metamaterials. Scientific Reports. 2016;6:34746

[45] Gric T, Hess O. Tunable surface waves at the interface separating different graphenedielectric composite hyperbolic metamaterials. Optics Express. 2017;25(10):11466-11476

[46] Li S, Jadidi MM, Murphy TE, Kumar G. Terahertz surface plasmon polaritons on a semiconductor surface structured with periodic V-grooves. Optics Express. 2013;21:7041-7049

[47] Kumar G, Li S, Jadidi MM, Murphy TE. Terahertz surface plasmon waveguide based on a one-dimensional array of silicon pillars. New Journal of Physics. 2013;15:085031

[48] Rusina A, Durach M, Nelson KA, Stockman MI. Nanoconcentration of terahertz radiation in plasmonic waveguides. Optics Express. 2008;16:18576-18589

[49] Shen L, Chen X, Yang T-J. Terahertz surface plasmon polaritons on periodically corrugated metal surfaces. Optics Express. 2008;16:3326-3333

[50] Gric T. Surface-plasmon-polaritons at the interface of nanostructured metamaterials. Progress in Electromagnetics Research. 2016;46:165-172

[51] Vassant S, Hugonin J-P, Marquier F, Greffet J-J. Berreman mode and epsilon near zero mode. Optics Express. 2012;20:23971-23977

[52] Vassant S, Archambault A, Marquier F, Pardo F, Gennser U, Cavanna A, Pelouard J, Greffet J-J. Epsilon-near-zero mode for active optoelectronic devices. Physical Review Letters. 2012;109:237401

[53] Campione S, Brener I, Marquier F. Theory of epsilon-near-zero modes in ultrathin films. Physical Review B. 2015;91:121408 
